\title{
Plants Grow Virtual Reality Simulation Technology Research
}

\author{
Qin Yuping, Wang Xiaojuan, Zhang Shuang \\ The Engineering \& Technical College of Chengdu \\ University of Technology \\ Leshan, china
}

\begin{abstract}
Virtual reality technology is an extremely important means in the modern teaching, it overcome the impact of conditions. Plants grow virtual reality simulation is in an extremely important part of virtual reality. This paper analyzes the $L$ system and the IFS system using Matlab complete the virtual reality simulation; Through the adjustment factor, realized the random tree and virtual reality scene simulation; provide the basis for plant growth condition simulation .
\end{abstract}

Keywords-Plant growth, Virtual reality, L system, IFS system

\section{INTRODUCTION}

Experiment is indispensable in technology teaching activities, and is extremely important segment. It not only can cultivate students' practical ability, also can improve students' ability to solve problems. In science teaching activities, the students' practical ability is mostly got by doing experiments. But not all schools are able to provide students with the necessary conditions of experimental required, for several reasons the practice curriculum design non-existing. Thus virtual reality simulation technology for the students practice ability cultivation of a very important method. It has image lifelike, users can computer through various channels to interact in virtual reality, gain hearing, vision and so on various aspects of satisfy. Thus reach confront wonderland feeling, meet the students' time teaching requirements.

Plants grow virtual reality simulation technology is simulated by computer technology plant growth in a development process. Simulated plant not only reflect the plant's growth process, morphological structure, with the growth of $3 \mathrm{~d}$ effects plant individual or group. Also can simulate environmental changes plant growth changes. Using this method not only can calculate plant growth model, also can get mutual parameters and artificial environment for the growth of a plant mutual parameters influence, in order to achieve the monitor on test data. Using this method to establish virtual farm in the system, so that the students can obtain essential agricultural management knowledge, can get the effect that traditional methods cannot get .

\section{PlaNT GROWTH SIMULATION}

In order to study the growth of plants rule, in the mid 1960 s, people started plant growth virtual reality simulation, and by establishing a mathematical model to simulate the process of plant physiology ecology, thus speculate in

\author{
Xiao Jing \\ Xu Zhou Air force College \\ XuZhou, China
}

different ecological environment, under control the growth of a plant for certain composite index. If the germination rate, leaf plant growth, and flowering, etc.

Fractal simulation method is a use of plant morphological structure of fractal nature produces plants graphics and image modeling method. It includes L system, IFS (iterative function) system, etc.

\section{A. system}

System is Lindenmayer 1968 American biologist for the modeling of biological forms and described the design method of plant configuration and growth. System is actually a string rewrite system. The string is explained as graphics, so as long as can generate a string, is also generating a graphics. From an initial string (called justice) notes for beginning, will create rules applied to the many times, and eventually produce a longer command string, and use it to drawing.

For system can use more complex graphics explanation, in addition to the analog plants branch topology outside, plus line length and the corner and so on geometric shapes. System of strings of symbols, also known as "turtle line graph" (turtle), namely, imagine a tortoise in the plane, the tortoise state crawling with triad (X, Y, D) says, including X and $\mathrm{Y}$ respectively represent the abscissa denotes and $y$-coordinate represent the current orientation, D. Make the delta is Angle incremental, $h$ is step length. This paper uses the symbols provisions and L system explanation: F: from the current position and move forward step by step length of drawing a line for $h$, at the same time; $G$ : moving forward from the current position for $h$, step length step, but don't draw lines; + : from the current direction anticlockwise a given Angle delta; - : from the current direction clockwise turn a given Angle delta; | : spin $180^{\circ}$; [: shall we do graph, turtle press in current state of the stack); : Pop, will graphics state to reset stack state, and remove the stack of content; A: records state direction; $\mathrm{Z}$ : record current position.

Simple branching structure representation for: set the initial formation yuan $\mathrm{W} \mathrm{F}$, rewrite rules $\mathrm{P}$ for: $\mathrm{FF}+[+\mathrm{F}-\mathrm{F}$ - F $]$ - $[-F+F-F]$. One $F$ for forward symbols. MATLAB2010a software is built using virtual reality modeling, the results as shown in figure:

Figure 1 iterations $n=2$ figure 2 iterations $n=3$

Simple drawings of trees branching structure like a primitive isn't outstanding, the backbone of forest shrub, in order to overcome the shortcomings of the branching structure, with empty instructions instead of complicated branch of the structure in format. Bring back the branch of empty instruction format for: a structure representation for 
generating yuan initial $\mathrm{X}$, rewrite $\mathrm{P} \mathrm{W}$ for: $\mathrm{FF}-[-\mathrm{X}+\mathrm{X}+\mathrm{X}]$ $+[+\mathrm{X}-\mathrm{X}-\mathrm{X}]$. One $\mathrm{X}$ is a forward symbols, when in use, is equivalent to $F$, need not when, is an empty instructions. When rewriting the rules only with $\mathrm{X}$ to make the string recursion. MATLAB2010a software is built using virtual reality modeling, the results as shown in figure:

\section{B. iterative function system (IFS)}

Iterative Function System (Iterated views are fractal rendering) was typical important method. Its use of certainty algorithm and the way of combination of random algorithm generating plant stem stem or leaf weapon.these charts. "Authenticity" refers to the rules to iterative are deterministic, they by a group of affine transform (such as etc) constitute; "Random" means the iterative process is uncertain, namely each time actually iteration which rule is random, a final to create graphics (plant shape charts), it must meet for collection equation. Formula means, randomly selected from an iterative rules iteration, then randomly once appoint one rule iteration time in, to repeat this process, finally generated limit graphics $\mathrm{M}$ is the request of plant shape charts.

In practical fractal graphs, often by random iterative generating plant leaves with infarction in the original IFS, namely, add a group of random Numbers. The leaves of the generated by three affine transformation and the corresponding probability vector decision. A two-dimensional affine transformation for the form

By setting the affine transformation parameters, using MATLAB2010a establishing virtual reality modeling, can generate fern leaves as shown in the diagram

\section{MODEL DEVELOPMENT}

The effect from analog plants with above ways to get graphics too simple, inflexible; The reality of plants than the front generated graphics will much more complicated. The connotation of by adding more genes in the reserves, or under the premise of main characteristics change some

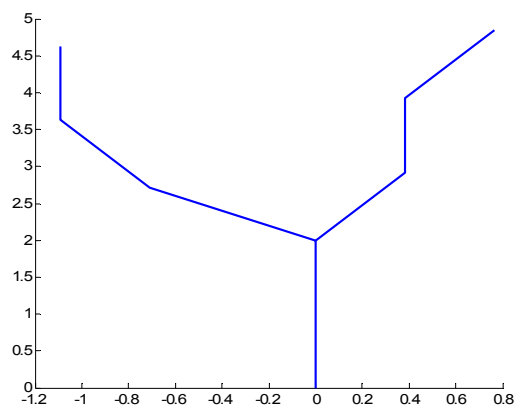

Figure 1. iterations $\mathrm{n}=2$ details (for example, will Angle multiplied by a greater than 0 to 1 random factor between when each iteration, such changes Angle to vivid realism,) to realize the more advanced some modeling. Figure 13 is random tree illustrations, figure 14 is virtual scene.

Figure 13 random tree graph and virtual environments

\section{CONCLUSION}

Plant virtual reality modeling system is using mathematical method to simulate the growth of a plant a virtual reality system. Using this system can be used to simulate environmental factors on the influence of plant growth process, on the other hand, we can get lots of traditional methods cannot get results. Through the analysis of L system and the principle of IFS system, use of Matlab simulation the plant's virtual reality simulation, and through the adjustment factor for the first time in Matlab, realization of virtual scene.

\section{REFERENCES}

[1] de Reffye P,Edelin C,Franqon J,et a1.Plants Models Faithful to Botanical Structure and Development[J].Computer Graphics, 1998,22(4):151-158.

[2] Guo Y, Li B G.New Advances in Virtual Plant Research[J].Chinese Science B,2001,46(11): 888-894.

[3] Prusinkiewicz P,Lindenmayer A,Hanan J.Developmental Models of Herbaceous Plants for Computer Imagery Purposes[J] Computer Graphics,988,22(4): 141-150.

[4] Room P M,Hanan J S.Prusinkicw P.Virtual Plants:New-242 Perspectives for Ecologists,Pathologists and Agricultural Scientists[J].Trends in Plant Science, 1996, 1(1): 33-38.

[5] Oppeheimer P.Real Time Design and Animation of Fractal Plants and Trees[C]Proceedings of SIGGRAPH.1986: 55-64.

[6] Lindenmay A.Mathematical Models for Cellular Interaction in Development Parts $1 \& \quad 11[\mathrm{~J}] . J$ Journal of Theoretical Biology, 1968,16(2):280-315.

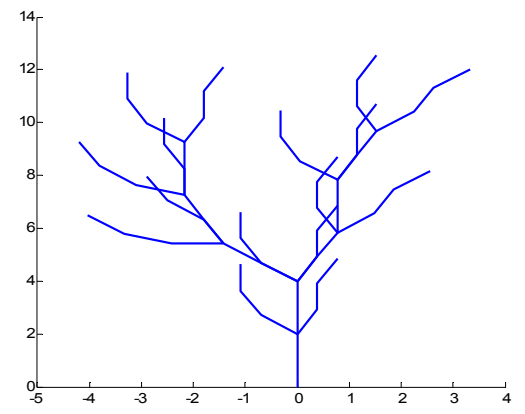

Figure 2. iterations $n=3$ 


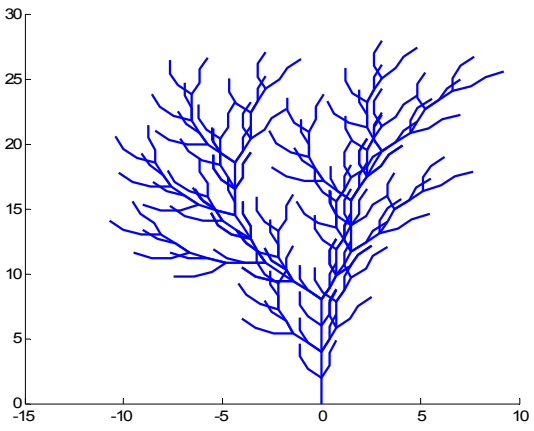

Figure 3. iterations $n=4$

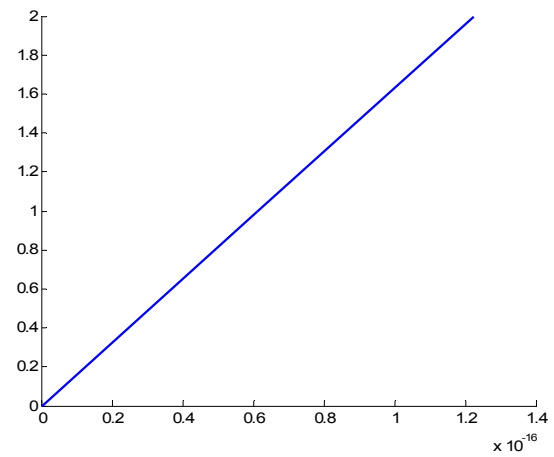

Figure 5. iterations $\mathrm{n}=2$

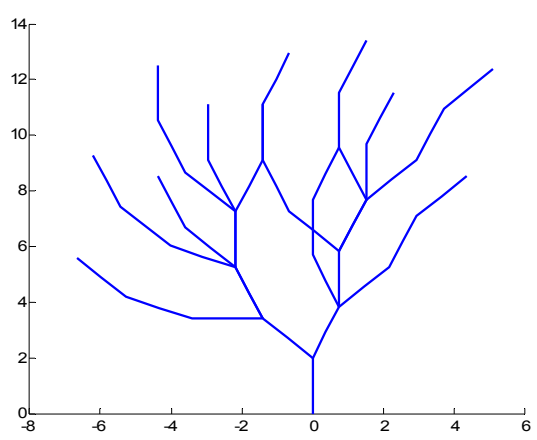

Figure 7. iterations $n=4$

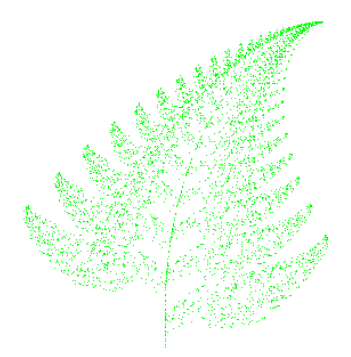

Figure 9. iterations $n=5000$

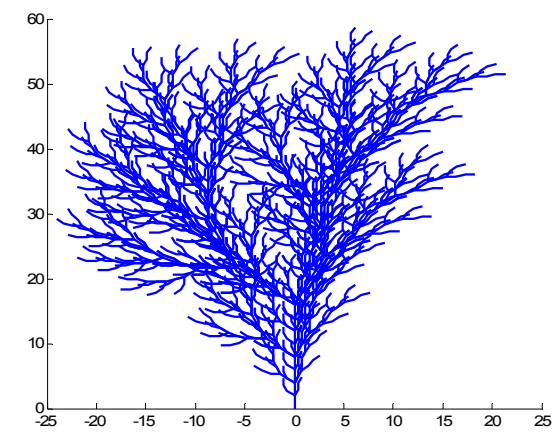

Figure 4. iterations $n=5$

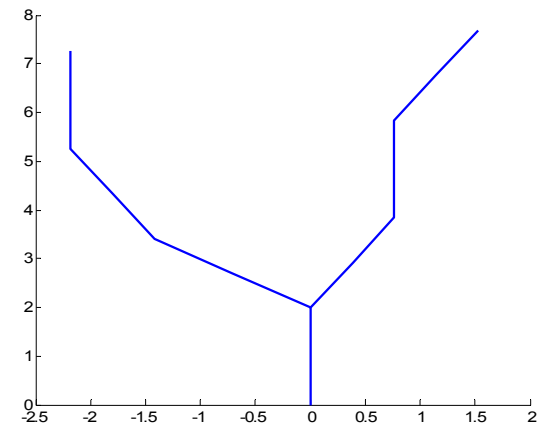

Figure 6. iterations $n=3$

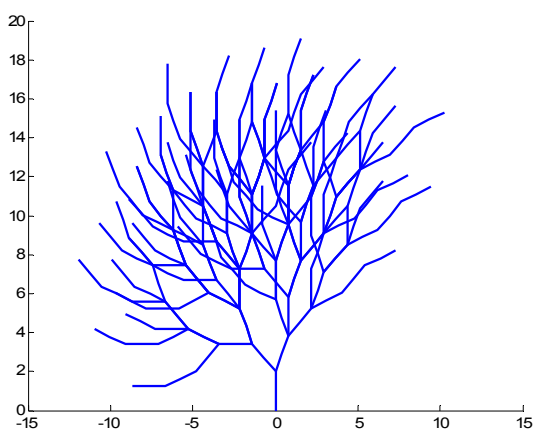

Figure 8. iterations $\mathrm{n}=5$

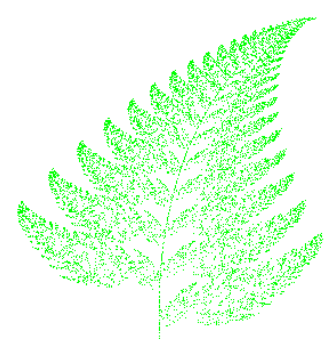

Figure 10 . iterations $n=10000$ 

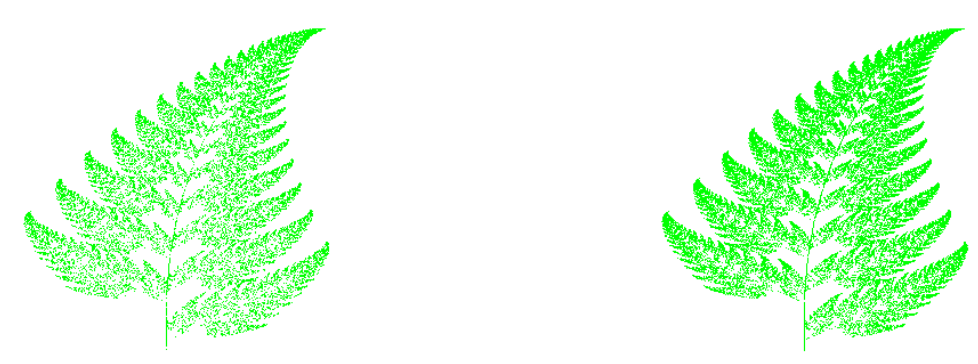

Figure 11. iterations $n=20000$

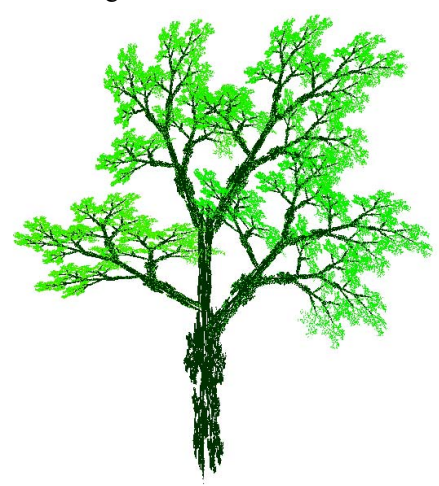

Figure 12. iterations $n=40000$

Figure 13. random tree

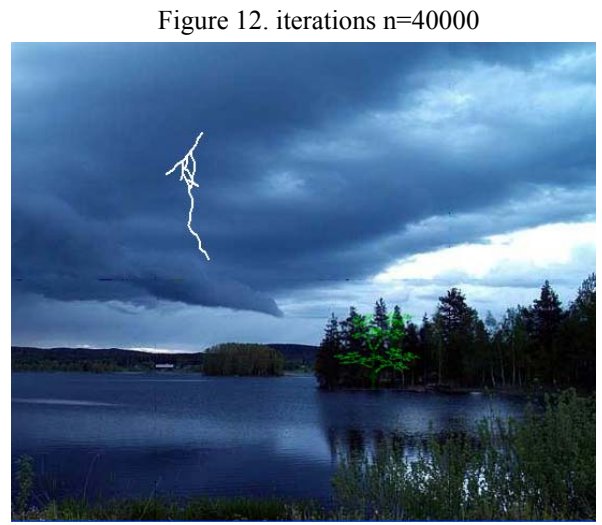

Figure 14. virtual environments 